\title{
Spatial evolutionary prisoner's dilemma game with three strategies and external constraints
}

\author{
György Szabó, ${ }^{1}$ Tibor Antal, ${ }^{2}$ Péter Szabó, ${ }^{3}$ and Michel Droz ${ }^{2}$ \\ ${ }^{1}$ Research Institute for Technical Physics and Materials Science \\ P.O.Box 49, H-1525 Budapest, Hungary \\ ${ }^{2}$ Department of Theoretical Physics, University of Geneva, 1211 Geneva 4, Switzerland \\ ${ }^{3}$ Department of Ecology, József Attila University, H-6721 Szeged, Egyetem u. 2, Hungary \\ September 17, 2018
}

\begin{abstract}
The emergency of mutual cooperation is studied in a spatially extended evolutionary prisoner's dilemma game in which the players are located on the sites of cubic lattices for dimensions $d=1,2$ and 3 . Each player can choose one of the three following strategies: cooperation $(C)$, defection $(D)$ or Tit for Tat $(T)$. During the evolutionary process the randomly chosen players adopt one of their neighboring strategies if the chosen neighbor has higher payoff. Moreover, an external constraint imposes that the players always cooperate with probability $p$. The stationary state phase diagram is computed by both using generalized mean-field approximations and Monte Carlo simulations. Nonequilibrium second order phase transitions associated with the extinction of one of the possible strategies are found and the corresponding critical exponents belong to the directed percolation universality class. It is shown that forcing externally the collaboration does not always produce the desired result.
\end{abstract}

PACS numbers: 02.50.-r, 05.50.+q, 87.23.Cc

\section{INTRODUCTION}

Evolutionary game theory has attracted a lot attention during the past years [1] 2 in human sciences, political sciences, biology and economics. In particular, the so-called evolutionary prisoner's dilemma game (PDG), which is a metaphor for the evolution of cooperation in populations of selfish individuals, has been minutely investigated [1 5 . In the original form of the PDG, only uniform populations with given strategies were considered. However, it was realized [1, 6 ] that new interesting phenomena can occur when the PDG was expanded in such a way that local contests in a d-dimensional space could take place (we shall use the abbreviation SPDG for such systems). It turns out that these spatially extended models are similar to the ones studied in nonequilibrium statistical physics. They may exhibit cooperative behavior resulting in phase transitions in the stationary state. Accordingly, it is very fruitful to study SPDG like models using the tools developed in the framework of nonequilibrium statistical physics.

In its simpler form, the PDG is a version of matrix games where the symmetric incomes of the two players depend on their simultaneous decisions, whether they wish to cooperate with the others or to defect. Each player wants to maximize his individual income. The highest individual payoff (the temptation to defect) can be reached by the defector against the cooperator receiving the lowest reward (sucker's payoff). The mutual cooperation results in the highest total payoff divided equally between the players. For mutual defections the players get a lower payoff exceeding the value of sucker's payoff. Two rational players will both defect because this choice provides the larger income independently of the partner's choice.

On the contrary, mutual cooperation dominates in economic and biological systems where the contestants interact frequently. In the iterated round-robin PDG, the players, knowing the previous decisions, have to choose between two options (defection and cooperation). For a given round the contestants can be classified according to the total individual payoffs they have obtained. Following the Darwinian selection principle, at each round the worst player will adopt the winner's strategy.

Extended numerical simulations have been performed

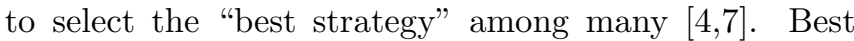
does not means that this strategy will always win a fight against another strategy, but means that it will obtain the highest payoff during a tournament during which it will have to fight against many opponents having different strategies. In [4 the highest payoff was obtained by the so-called "Tit for Tat" $(T)$ strategy which cooperates in the first round and then always repeats his coplayer's previous decision. The main characteristics of this strategy (never defect first, react to the defection of the opponent and forgiveness) are crucial ingredients to sustain the mutual cooperation against the defectors. In particular, extensive simulations (see [3] for a summary) for the case in which the players adopt one of the two following strategies: $C$ (cooperate unconditionally) or $D$ (defect unconditionally) have shown that the cooperators were disappearing in the stationary state. The introduction of some $T$ 's strategies has an important effect. For short times, the D's population increases while the C's one decreases, leading to the decrease of D's payoffs. As a consequence, the T's can invade the D's population.

In order to study the spatial effects Nowak and May [6] have introduced an SPDG consisting of a two-state cel- 
lular automaton. The players are located on a regular lattice in a d-dimensional space and can adopt the C's or D's strategy. Each player is fighting with the individuals belonging to a given neighborhood. The player's strategies are upgraded simultaneously in discrete time steps according to the following rule: each player adopts the best strategy found in his neighborhood. This model exhibits a rich variety of spatial and temporal patterns as a function of the payoff $b$ characterizing the temptation to defect. Other SPDG models have also been investigated [8 10]. In particular Killingback and Doebeli [11 have shown that "Pavlov" like strategies can be even more efficient than "Tit for Tat" in some circumstances.

Nowak et al. 12 have also extended the above analysis by allowing stochasticity (irrational choices) during the evolutionary process. The degree of stochasticity is governed by a parameter called $m$, and in the limit $m \rightarrow \infty$, one recovers the deterministic case. According to the value of $b$, several stationary states are possible.

In some related models [13], Szabó and Tőke have shown that the different stationary state phases were separated by second order nonequilibrium phase transitions lines. The associated critical exponents belongs to the directed percolation (DP) universality class 13,14.

Both the importance of the presence of the T's in the PDG and the richness associated with the spatially extended aspect motivated us to study a new class of SPDG. In the present work, we study a novel aspect of the SPDG, namely what is happening when the cooperators are enforced by some external constraints. More explicitly, we consider a SPDG with the three strategies $D, C$, and $T$ and investigate the effects of random adoption (or forcing) of $C$ strategies. This new effect can be interpreted as an attempt by a government or by any other organization to enforce the cooperation among individuals by forcing some of them, chosen randomly with a given probability $p$, to cooperate. A different interpretation can also be given. Among the player's community, some old players resign and are replaced by younger ones having a different educational background making them more open to collaborate. As we shall see the effects of this external constraint are rather surprising. Indeed, in dimensions 1,2 or 3 , the presence of the constraint reduces the cooperation if $p$ is less than a given threshold value $p_{c}$ depending upon the dimensionality of the system. The cooperation is enforced only if the constraint is strong enough, i.e. if $p>p_{c}$.

The nonequilibrium second order phase transitions describing the extinction of one of the possible strategies are found to belong to the directed percolation universality class.

The paper is organized as follows. The model is defined in section II. Its properties are analyzed in mean-field approximation in section III. The properties of the model without constraint are discussed in section IV. The model with constraint is studied in section $\mathrm{V}$, both in mean-field approximation and by Monte Carlo (MC) simulations, for respectively 1, 2 and 3 dimensions. Finally, conclusions are drawn in section VI.

\section{THE MODEL}

We consider a SPDG model in which the players are located on the sites of a $d$-dimensional cubic lattice of linear size $L$ where periodic boundary conditions are assumed. Each player can adopt one of the three following strategies $D$ (defects), $C$ (cooperates) or $T$ (Tit for Tat) and interacts with its $2 d$ nearest neighbors. The total payoff of a given player is the sum of the payoffs coming from the interaction with all its nearest neighbors. We use an extension (including the Tit for Tat strategy) of the payoff matrix used by Nowak and May [6]. The individual payoffs for the players $P_{1}$ and $P_{2}$ as a function of their strategies are given in Table 1 . The only free parameter $b(1<b<2)$ measures the temptation to defect. Note that the above payoff matrix does not take into account that the $T$ players always try to cooperate with D's during the first round. Thus these values are considered as the averaged (or in this present case stabilized) payoffs.

\begin{tabular}{|c|c|c|c|}
\hline \begin{tabular}{|l|l}
$P_{1} \backslash P_{2}$ \\
\end{tabular} & $D$ & $C$ & $\bar{T}$ \\
\hline$D$ & $0 \backslash 0$ & $b \backslash 0$ & $0 \backslash 0$ \\
\hline $\bar{C}$ & $0 \backslash b$ & $1 \backslash 1$ & $1 \backslash 1$ \\
\hline $\bar{T}$ & $0 \backslash 0$ & $11 \backslash 1$ & 1 \\
\hline
\end{tabular}

Table 1: Payoff matrix of the model

It is legitimate to use this payoff matrix providing that the strategy adoption is rare comparing to the frequency of the game. It makes the simulation simpler and more efficient. Notice that similar payoff matrices can be obtained when substituting some other "nice" strategy (as Pavlov which cooperates in the first round for example, a strategy which is nevertheless less efficient than $\mathrm{T}$ in this case) 3, 4, for $T$.

For the non constrained case $(p=0)$, the system evolves in discrete time according to the following $\mathrm{MC}$ process. Starting from a random initial state, a site is chosen randomly. This site updates its strategy by first selecting randomly one of its nearest neighbor and second, by adopting the strategy of this nearest neighbor only if it is having a higher payoff. A MC steps consists in updating each lattice site once, on the average.

In the constrained case, at each time step, each player is forced to adopt the cooperative strategy $C$ with a probability $p$.

Thus the dynamics of the constrained model is the following.

- One chooses randomly one player.

- With probability $p$ this player adopts the $C$ strategy. 
- With probability $(1-p)$ the player searches for a better strategy according to the procedure described above.

- The players update their payoffs.

The model is characterized by three free parameters: $b, p, d$.

It is hopeless to find exact analytical solutions for such models. Accordingly, we shall first study them in the framework of mean-field like approximations and then investigate them by numerical simulations.

\section{MEAN-FIELD LIKE APPROXIMATIONS}

The simplest mean-field approximation consists in neglecting all the spatial correlations in the systems. This amount to consider a model in which for each player, the partners are chosen randomly in the system instead of being restricted to a particular neighborhood. Each player interacts with the same number of counterparts. The dimensionality of the system plays no role. The simplest mean-field like approximations have been successfully used previously for similar problems and more details concerning this approximative scheme can be found in textbooks [1, 2, 2, 5].

Within this approximation, the dynamics of the system is completely described by the time dependent concentrations:

$$
c_{\alpha}(t)=\left\langle N_{\alpha}(t)\right\rangle / L^{d}, \quad(\alpha=C, D, T)
$$

where $N_{\alpha}(t)$ is the number of players with strategy $\alpha$ at time $t$. These concentrations satisfy the normalization condition $c_{D}+c_{C}+c_{T}=1$.

According to Table 1 the average payoffs for each strategy are:

$$
m_{D}=b c_{C}, \quad m_{C}=c_{C}+c_{T}, m_{T}=c_{C}+c_{T} .
$$

Notice that the $C$ and $T$ strategies have the same payoffs, therefore no strategy exchange will occur among them.

Following the evolutionary rules given in Sec. II, the concentrations $c_{\alpha}(t)$ obey the following equations of motion:

$$
\begin{aligned}
& \dot{c}_{D}=-p c_{D} \mp(1-p) c_{D}\left(c_{C}+c_{T}\right), \\
& \dot{c}_{C}=+p\left(c_{D}+c_{T}\right) \pm(1-p) c_{D} c_{C}, \\
& \dot{c}_{T}=-p c_{T} \pm(1-p) c_{D} c_{T},
\end{aligned}
$$

where the upper and lower signs refer respectively to the cases when strategy $D$ is dominated by $C$ and $T\left(m_{D}<\right.$ $\left.m_{C}=m_{T}\right)$, and when $D$ dominates $C$ and $T\left(m_{D}>\right.$ $\left.m_{C}=m_{T}\right)$.

The numerical integration of the above equations of motions leads, for several values of $p$, to the flow diagrams shown in Fig. 1.
The quantities represented on the vertical and horizontal axes are respectively $c_{D}$ and $c_{C}-c_{T}$. The upper corner of the triangle corresponds to the state of $c_{D}=1$, while the lower left and right corners describe respectively homogeneous states with $c_{T}=1$ and $c_{C}=1$.
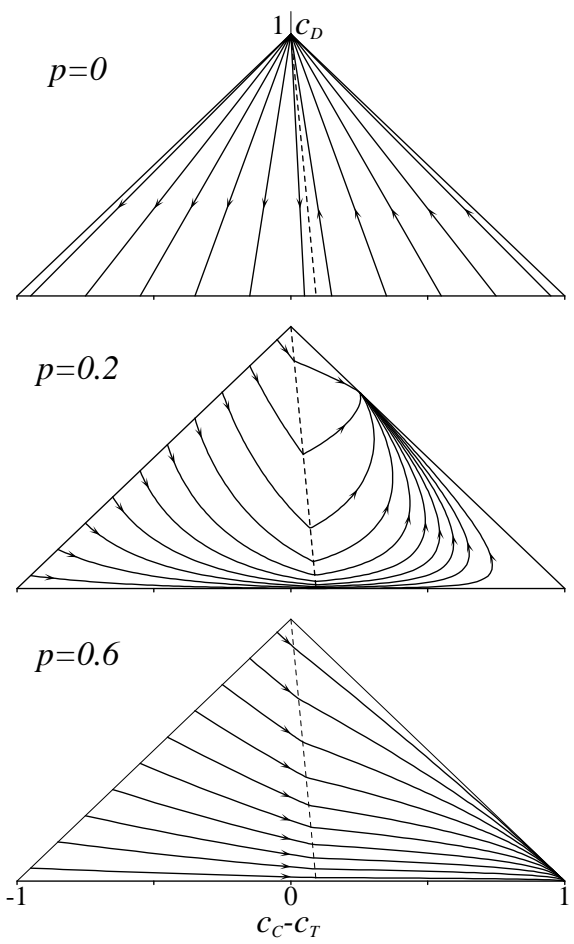

FIG. 1. Trajectories in the two-dimensional phase space for three different values of $p$ as indicated in the figures. The dashed lines divide the phase spaces into two regions: on their left (right) hand side the payoff of the $D$ strategy is lower (higher) than those of the $C$ and $T$ ones.

For $0<p<1 / 2$ the stationary state solution of the above equations is:

$$
c_{D}=\frac{1-2 p}{1-p} ; \quad c_{C}=\frac{p}{1-p} ; \quad c_{T}=0
$$

while for $p>1 / 2$ the system goes to the adsorbing state $\left(c_{C}=1\right.$ and $\left.c_{D}=c_{T}=0\right)$. Surprising the $T$ strategy extincts if $p>0$.

Without constraints, i.e. for $p=0$, the system tends either to a homogeneous $D$ adsorbing state $\left(c_{D}=1\right)$ or to a mixed state of $C$ and $T$ strategies $\left(c_{D}=0\right)$ depending on the initial conditions. Note that the above stationary states are independent of the value of $b$. Strikingly different behaviors will be observed beyond this simplest mean-field approximation when the local fluctuations and short-range correlations are taken into account as we shall see in the following sections.

More elaborated mean-field like approximations can be devised. The basic idea is to take explicitly into account some of the spatial correlations by computing the probability of appearance of all the possible configurations 
of a small cluster containing $n$ sites. In one dimension, one considers a cluster of $n$ consecutive lattice sites. The equations of motion for these probabilities follows from the evolution rules of the system. Details concerning the one-dimensional case are given in [15]. Note that already the one site mean-field approximation $(n=1)$ differs from the simple mean-field solution given above. In higher dimensions pair or square mean-field like approximations have been used, and a detailed description of these methods can be found in 13, 16. Note that now, the predictions of these approximations depends upon the dimension of the system. Accordingly, the cases $d=1,2$ and 3 will be discussed in different subsections.

\section{MODEL WITHOUT EXTERNAL CONSTRAINT}

Let us start by considering the case with no external constraint, i.e. $p=0$. In this case, the dynamic is simple: a given player adopts the strategy of one of his randomly chosen neighbor providing that this neighbor has a higher payoff. As we shall see, the only stationary states are either always trivial cooperator like $(C$ or $C-T)$ or a pure defector one $(D)$.

When the random initial state is made of only cooperators and defectors, one founds that, both in $d=1,2$ and 3 dimensions, the stationary state of the system is a pure defector one.

The reason in $d=1$ is simply that a $D$ player has always a higher payoff than a neighboring $C$ player.

In $d=2$ several configurations should be analyzed. The most favorable situation for $C$ to win is when it is adjacent to a flat $C-D$ interface. However, if this interface has an irregularity then the $D$ 's can invade the sea of $C$ players. Indeed, a new born $C$ in the $D$ half-plane is always weaker than the $D$ 's at the interface and thus, such $C$ 's disappears sooner or later. However, at a given time and with some finite probability, two next nearest neighbor $C$ players could be present in the $D$ half-plane. It results a payoff $3 b$ for the $D$ player squeezed between three $C$ and this $D$ can now invade the $C$ 's and sweep off one layer of them independently of the value of $b$.

The $d=3$ case is very similar, but the process is slower. Indeed, four nearest neighbors of a $D$ player (called $D_{1}$ ) are necessary to be invaded by the $C$ 's if the value of $b$ is close to 1 . Once it has occured, $D_{1}$ is strong enough to cross the interface and then destroys all the $C$ 's.

For a large system with initially a finite proportion of $T$ players, the stationary state is always a cooperator like state $(T-C)$. This asymptotic behavior can be easily understood in the one dimensional case. Let us suppose that there are four neighboring $T(T T T T)$ in the initial state of a $1 \mathrm{~d}$ system, (this is practically always the case in a sufficiently large system with a finite probability to have some $T$ 's in the initial state), then they kill all of the $D$ 's. Even in the worst case two $D$ 's could invade into to the $T$ population, $(C D D T T D D C)$, but then the central $T$ 's become stronger (payoff $=1$ ) then the $D$ 's (payoff $=0$ ). The $D$ 's kill all of the neighboring $C$ 's, and even these two $T$ 's can invade the whole $D$ area.

This argument can be extended to $d$ dimensions. A cube of linear size 4, made of $T$ players is enough to guarantee the cooperation in the stationary state. The reason is that the $D$ 's surrounding this cube cannot destroy the central $T$ players inside a cube of linear size 2 . Indeed, the central T's have always $d T$ 's neighbors to cooperate with (payoff $=d$ ), but the neighboring $D$ 's can only have one $C$ neighbor (payoff $\leq b<d$ if $d \geq 2$ ).

\section{MODEL WITH EXTERNAL CONSTRAINT}

We now consider the case in which the players are located on the sites of a d-dimensional cubic lattice in the presence of an external constraint imposing to each player to choose the $C$ strategy with probability $p$. The cases $d=1,2$ and 3 will be considered.

\section{A. One-dimensional system}

In the one-dimensional model the players located on the sites of a chain interact with their two nearest neighbors. It is easy to see that the dynamics is independent of the value of the parameter $b$ in its domain of definition.

A systematic MC analysis of the stationary states was performed by varying the value of $p$ for different system sizes between $L=32$ to $L=16384$. Our simulations show that the T's strategy extincts for all values of $p$. However, as a function of $p$, the stationary state can be either a symbiosis of $C$ and $D$ strategies or a pure $C$ state as shown in Fig. 2. For very small values of $p$, one has a pure $C$ stationary state $\left(c_{C}=1\right)$ but, when $p$ reaches the value $p_{1}$, a first transition occurs to a stationary state in which the two strategies $D$ and $C$ coexist. At $p=p_{c 2}>$ $p_{1}$ the system undergoes a second continuous transition from the $C-D$ stationary state to the pure absorbing $C$ state.

The transition at $p_{c 2}$ is easy to understand. In one dimension and when only $C$ and $D$ strategies are present our model is equivalent to the contact process (CP) which was originally introduced as a simple model for epidemics 17]. In the $\mathrm{CP}$ a particle (sick person) can disappear at rate 1 , and an empty site (healthy person) can become occupied with rate $\lambda z / 2$, where $\lambda$ is the control parameter and $z$ is the number of particles in the neighborhood of the empty site. In our model the $D$ strategy, which in $d=1$ is always better than the $C$ one, plays the role of the sick persons and the $C$ strategy, which can only be created in the system through the constraint, corresponds to the healthy individuals. 


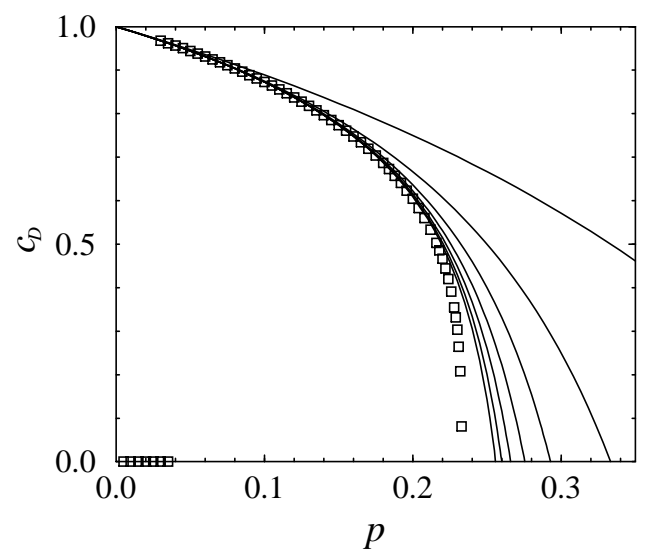

FIG. 2. The concentration of strategy $D$ as a function of $p$ in the one-dimensional system. The open squares are the MC data obtained for $L=16384$. The solid lines represent the results of $n$-point approximations $(n=1, \ldots, 7$ from right to left).

Starting from a $C-D-T$ initial state and after the extinction of $T$ 's the possible stationary states are the pure $C$ or a $C-D$ states. The $C-D$ state corresponds to the steady state of the CP as well. For $p$ around $p_{c 2}$, the $T$ 's disappear rapidly and thus do not affect the extinction of the $D$ 's. Hence the second transition to the absorbing state corresponds to the CP's one. This transition occurs at $p_{c 2}=1 /\left(1+\lambda_{c}\right)=0.23267$ [18] and is believed to belong to the DP universality class [19,20].

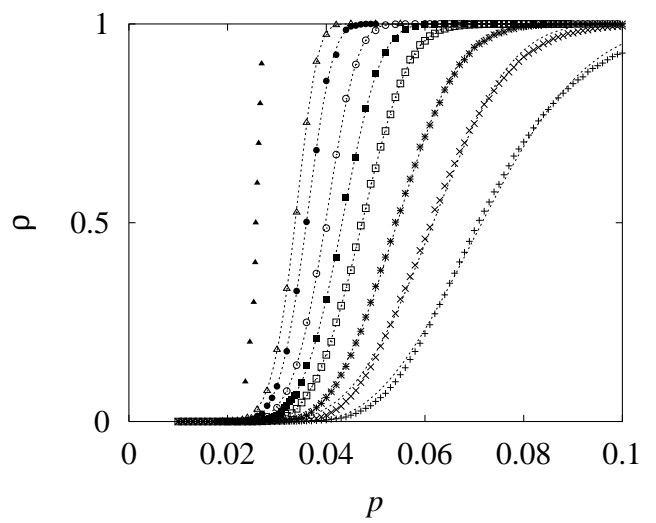

FIG. 3. The probability, $\rho_{L}(p)$, of reaching the $C$ - $D$ state for different system sizes (from right to left $L=50,100$, 200, 500, 1000, 2000, 5000, 10000 and the interpolation for $L=\infty)$. The dotted lines represent a fit described in the text.

The behavior of the first transition at small $p$ 's is much less clear. It turns out that for our model two characteristic parameters $p_{\alpha}(L)$ and $p_{\beta}(L)$, depending on the system size $L$, can be introduced. For $p<p_{\alpha}(L)$ the stationary state is always the pure $C$ state while for $p>p_{\beta}(L)$ the system evolves to a $C$ - $D$ coexistence phase, which is a steady state of the CP. For $p_{1}(L)<p<p_{2}(L)$, the system can evolves towards one of the two possible sta- tionary states, depending on the particular realization of the random numbers and on the initial state.

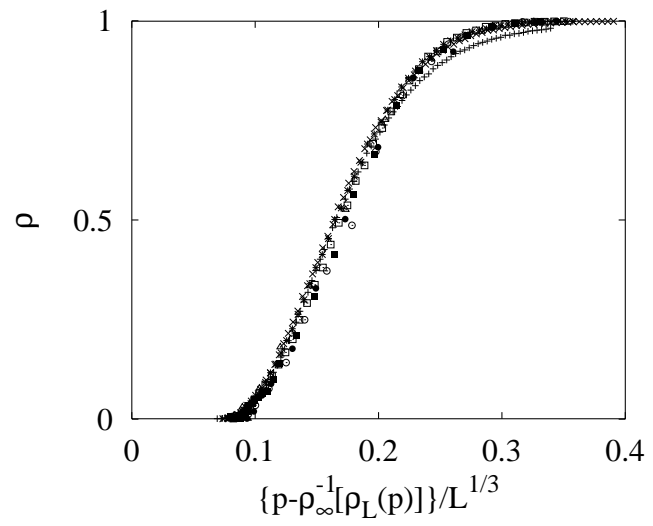

FIG. 4. The probability $\rho$ as a function of the scaled parameter. The symbols represents the different system sizes as in Fig. 3 .

The probability, $\rho_{L}(p)$, of reaching the $C$ - $D$ state has been investigated numerically. The data are plotted in Fig. 3 and can be well fitted by a function of the type $1-\exp \left(-c_{1}\left(p-p_{0}\right)^{c_{2}}\right)$, where $c_{1}, c_{2}$ and $p_{0}$ are fitting parameters. The limiting function $\rho_{\infty}(p)$ was obtained by using usual finite size scaling methods. As shown in Fig. 14, the functions $\rho_{L}(p)$ collapse on a single curve if one plots $\rho$ as a function of $\left\{p-\rho_{\infty}^{-1}\left[\rho_{L}(p)\right]\right\} / L^{1 / 3}$. This shows that $\rho_{\infty}(p)$ do not collapse to zero, hence the phase transition takes place at a finite value of $p \simeq 0.025$.

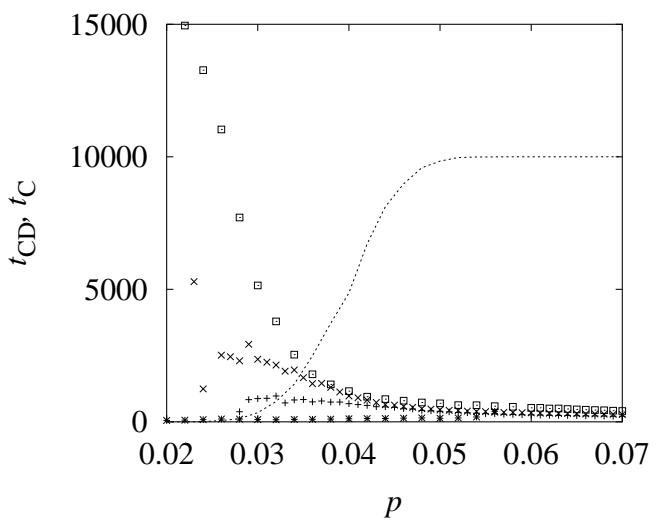

FIG. 5. The respective times $t_{C}(L=2000(*))$ and $t_{C D}(L=200(+), 500(\times), 2000(\square))$ needed to reach the $C$ and the $C-D$ stationary states. For comparison, $10000 \times \rho_{L=2000}(p)$ is also presented with dotted line.

The respective times $t_{C}$ and $t_{C D}$ needed to reach the pure $C$ and the $C-D$ stationary states have also been investigated. Both times show a singular behavior (see Fig. 5). Unfortunately, we were not able to find a reasonable scaling fit for those data. However, it is obvious from the simulations, that $t_{C D}$ exhibits a much stronger singularity than $t_{C}$. Hence, for a large system, the time 
needed to reach the $C$ - $D$ state is much larger than the one needed to reach the $C$ state, even if the system evolves to the $C$ - $D$ state more frequently.

Beside the MC simulations, the properties of the system were also investigated using generalized mean-field approximations. Assuming the coexistence of all the three strategies these calculations can be performed numerically on clusters of sizes as large as $n=4$. Contrary to the MC results (see Fig. 2) these approximations predicted the existence of a $C-D-T$ state at small $p$ values. As far as the T's are concerned, we observed that the maximum value of $c_{T}$ is decreasing when the cluster sizes $n$ were increasing, providing us a trend for the extinction of the T's.

The contradiction between the present mean-field and $\mathrm{MC}$ results refers to the importance of (interfacial) invasion phenomena detailed later on.

Allowing only two strategies $(C$ and $D)$ the above mean-field analysis can be performed for larger cluster sizes up to $n=7$. The results are compared with the MC ones in Fig. 2. As expected, the accuracy of the generalized mean-field method increases with the cluster size $n$. The extrapolation of the results obtained for finite values of $n(n=1, \ldots, 7)$ leads to a critical value $p_{c 2}^{(M F)} \simeq 0.235$. The quality of this approximative scheme can be estimated by comparing the value of $p_{c 2}^{(M F)}$ with the best known numerical value $p_{c 2}=0.23267$ [18].

In order to understand the behavior of the model around the first transition it is interesting to examine the time evolution of the system in its transient regime. As illustrated in Fig. 6, one can observe a domain growth process controlled by cyclic invasions [21]. This picture suggests that the most relevant aspect of the dynamics is the collision between the fronts separating different strategies.

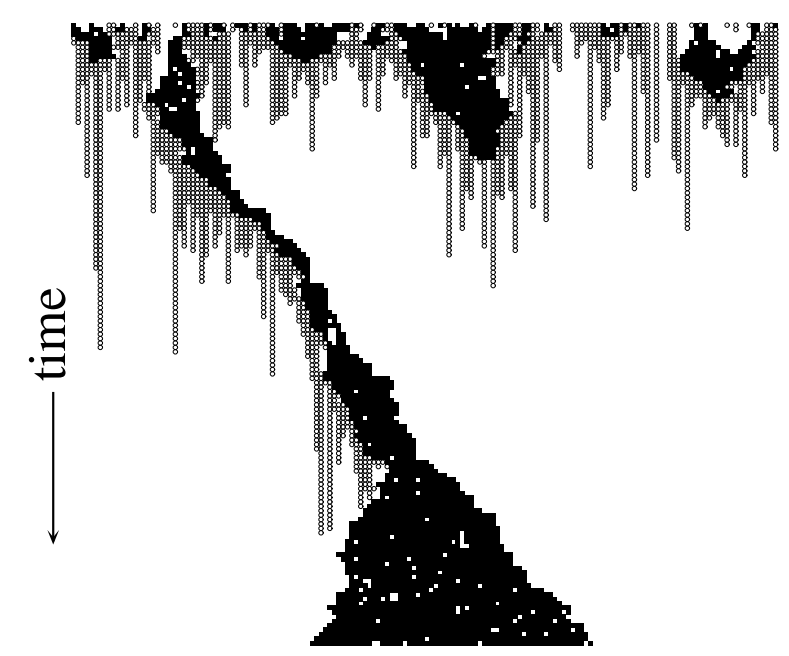

FIG. 6. Evolution of strategy distribution for $p=0.04$ starting from a random initial state. The $k$-th row shows the positions of the $D$ (closed squares), $C$ (empty), and $T$ (open circle) strategies at the time $2 k$ measured in MC steps.
Let us first investigate the motion of a front separating a cluster of $C$ from a $D$ one. The $D$ domains contain some $C$ sites coming from the external constraint. However, the life times of the $C$ 's are very short $[\sim(1-p)]$. Within a $D$ domain the average $C$ concentration is equal to that of the $\mathrm{CP}$ and can be well approximated by the simple mean-field approach as we have seen above. The $D$ strategy invades the territory of the $C$ one (absorbing state). Due to the reflection symmetry the invasion front can move to the left or to the right with the same absolute value of the velocity. To first order in $p$ in the limit $p \rightarrow 0$, the absolute value of the average invasion velocity can be estimated by using the configuration probabilities given for $n=1$. This calculation yields

$$
v=\frac{1-3 p}{2} .
$$

Let us now consider the invasion of the $T$ strategy into the $D$ one. Inside a territory which has been invaded by the $T$ strategy, the $C$ strategy is setting up with a probability $p$. As both $C$ 's and T's have the same payoff, no adaptation of strategies occur between them. Assuming that this invading front travels with a constant velocity $u$, the probability of having a $C$ player at a site $k$ steps behind the front is $1-e^{-p \tau(k)} . \tau(k)=(k+1 / 2) / u$ is the averaged time it takes to the front to move over a distance of $k$. Thus, it follows that, to leading order in $p$,

$$
u=\frac{1-11 p}{2} .
$$

We note that above approximations lead to a velocity for the $D \rightarrow C$ front which is larger than the one for the $T \rightarrow D$. This prediction can be compared with the results of the MC simulations (see Fig. 7). For the $D \rightarrow$ $C$ front, the agreement is very good if $p<0.1$, while for the $T \rightarrow D$ case, the approximation reproduces well only the linear part near the origin.

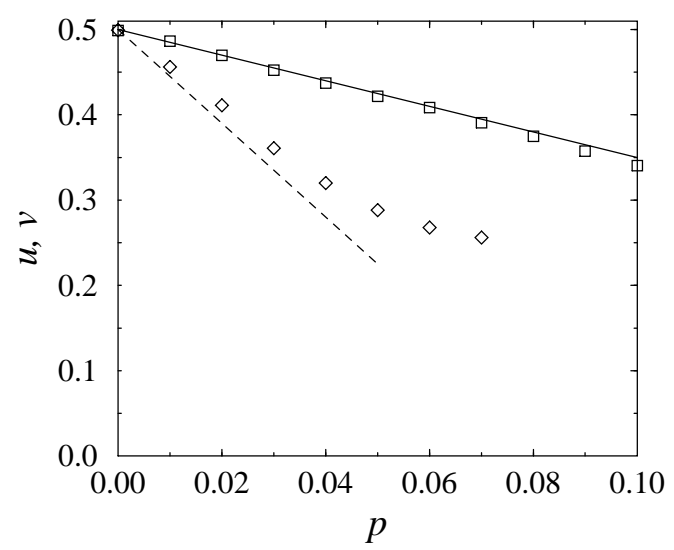

FIG. 7. Velocities of $D \rightarrow C$ (open squares) and $T \rightarrow D$ (diamonds) invasions as a function of $p$. The solid and dashed lines indicate the analytical predictions in linear approximation. 
Another consequence of the external constraint concerns the life-time of the $T$ clusters. According to the above picture, the probability that a $T$ cluster dyes out during the time evolution can be approximated by:

$$
P_{T}=\prod_{k=0}^{\infty}[1-\exp (-p(k+1 / 2) / u)] .
$$

The inverse of $P_{T}$ can be interpreted as the average life time $\tau_{T}$ of an invading $T$ cluster. Substituting an integral for the infinite sum appearing in the logarithm of the above expression leads to:

$$
\tau_{T} \simeq \exp \left(\frac{\pi^{2} u}{6 p}\right)=\exp \left(\pi^{2} \frac{1-11 p}{12 p}\right) .
$$

This expression shows a fast increase of the life-time when $p$ is decreasing. For example, a $T$ cluster dyes out in about $10^{5}$ MC steps (MCS) if $p=0.04$. This estimated life-time is significantly larger than those found by MC simulations $\left[\tau_{T}^{(M C)}(p=0.04) \approx 800 \mathrm{MCS}\right]$ as indicated in Fig. 6. The $p$-dependence of the MC data can be well approximated by the function $\tau_{T}=3.26 \exp (0.226 / p)$ within the region $0.025<p<0.08$ we could study. The large discrepancy between the mean-field and $\mathrm{MC}$ results refers to the enhanced role of the velocity fluctuations.

Starting from a random initial state spatially separated, domains are rapidly formed. Then two different situations can occur. First, $T$ clusters are present one both ends of each $D$ clusters leading to a fast extinction of $D$ 's. Accordingly, the system evolves to a pure $C$ state. Second, after some time the system reaches a state characterized by the presence of only one $D$ cluster, having a $T$ island at only one of its ends, in a sea of $C$. As the $D \rightarrow C$ invasion is faster than the $T \rightarrow D$, the $T$ 's can never destroy the $D$ 's and due to the finite life-time of the $T$ cluster the stationary state is a $C$ - $D$ coexisting one. The exponential increase of the life-time of this $T$ cluster as $p \rightarrow 0$ explains the singular behavior observed in $t_{C D}$.

For $p<p_{1}(L)$ the life-time of the $T$ players are so long that all the $D$ clusters are surrounded by T's. Accordingly, the first scenario described above is always present, leading to a pure $C$ state. In the contrary, for $p>p_{2}(L)$ the short lifetime of $T$ 's insures that the $T$ 's disappear rapidly, allowing for the growth of the $D$ clusters. As a consequence the stationary state is a $C-D$ one.

\section{B. Two-dimensional system}

The players are located on the sites of a twodimensional square lattice. According to the payoff matrix (see Table 1), two ranges of values of $b$ have now to be distinguished, namely, $1<b<3 / 2$ and $3 / 2<b<2$. For any value of $b$ in one of those ranges the dynamics is the same.

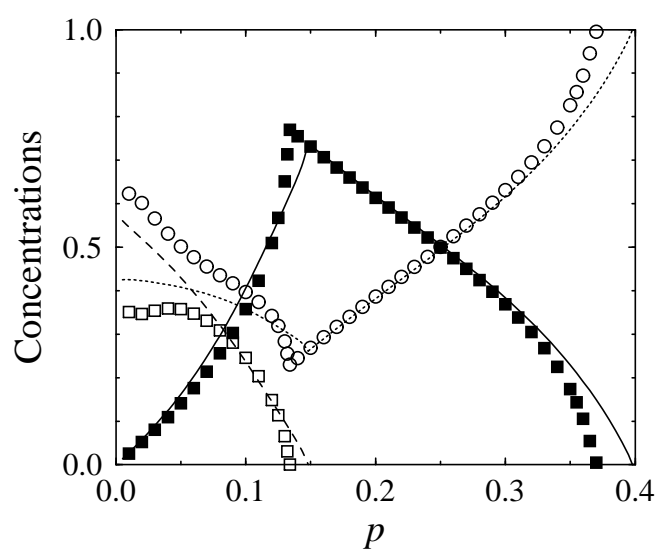

FIG. 8. Stationary state densities of $D$ (closed squares), $C$ (open circles) and $T$ (open squares) strategies as a function of $p$ as obtained by MC simulation of a system of size $512 \times 512$ for $3 / 2<b<2$. The solid $(D)$, dotted $(C)$ and long-dashed $(T)$ lines indicate the predictions of the square mean-field like approximation.

Let us first consider the simulations performed for the value $3 / 2<b<2$. The situation is summarized in Fig. 8, in which the stationary values of the strategy concentrations are plotted as a function of $p$. For $p<p_{c 1}^{(M C)}=0.1329(1)$, the system reaches a stationary state in which the three strategies $C, D$ and $T$ coexist. Here it is worth mentioning, that for small $p$ values $(p \lesssim 0.03)$ the finite system can reach the absorbing state if the initial state has been chosen randomly. Below this size-dependent threshold value the three-strategy state can be formed and sustained by slow decreasing of $p$ during the simulation. In this case the extinction of $T$ 's and $D$ 's is a consequence of fluctuations (detailed later on) and the coexistence of the three strategies is considered as the real stationary state in the limit $L \rightarrow \infty$. The simulations performed for $p \geq 0.005$ show that $c_{D}$ decreases linearly with $p$ when $p \rightarrow 0$.

When $p_{c 1}^{(M C)}<p<p_{c 2}^{(M C)}=0.3671(1)$ only the strategies $C$ and $D$ survive. Finally, when $p>p_{c 2}^{M C}$ the system reaches a pure $C$ absorbing state.

The phase diagram obtained by numerical simulations can be compared with the ones obtained using the extended mean-field approximation described in Sec. III. At the level of pair approximation, one finds $p_{c 1}^{(\text {pair }}=$ 0.1704 and $p_{c 2}^{(\text {pair })}=0.4236$, while for the square meanfield approximation one finds $p_{c 1}^{(s q)}=0.1482$ and $p_{c 2}^{(s q)}=$ 0.3980. These latest results are plotted in Fig. 8. They compare well with the $\mathrm{MC}$ values given above.

Some complementary information can be obtained by studying the concentration fluctuations defined as:

$$
\chi_{\alpha}=L^{d}\left\langle\left(N_{\alpha} / L^{d}-c_{\alpha}\right)^{2}\right\rangle, \quad(\alpha=C, D, T) .
$$

When $p \rightarrow 0$, the concentration fluctuations $\chi_{C}$ and $\chi_{T}$ are diverging while $\chi_{D}$ remains regular as shown in Fig. 9 . However, the sizes of the systems investigated were not 
large enough to conclude that $\chi_{C}$ and $\chi_{T}$ are diverging as power laws of $p$ in the limit $p \rightarrow 0$.

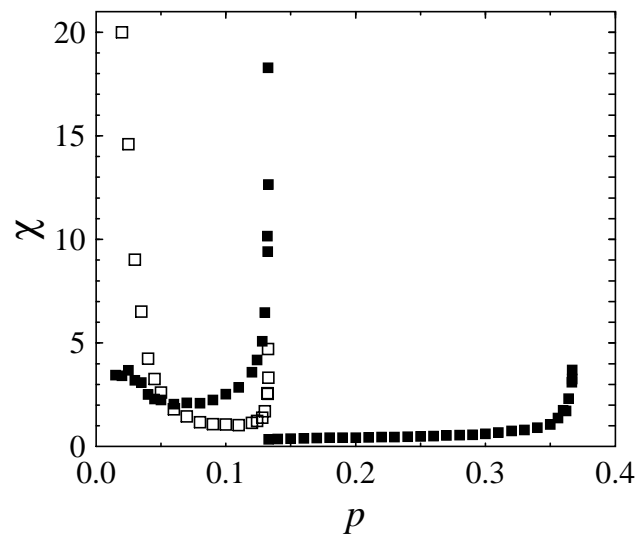

FIG. 9. Concentration fluctuations for the $D$ (closed squares) and $\mathrm{T}$ (open squares) strategies for a $512 \times 512$ system.

Moreover, the simulations suggest that for the stationary state in which the three strategies coexist, the typical size of $T$ domains (as well as their persistence time) is proportional to $1 / p$. Similar behavior was found in the forest fire models 22 introduced by Bak et al. 23. to study the self-organized criticality.

The points $p_{c 1}$ and $p_{c 2}$ are critical points where a second order nonequilibrium phase transition takes place. Indeed, the $T$ concentration vanishes at $p_{c 1}$ as $c_{T} \sim$ $\left(p_{c 1}-p\right)^{\beta_{1}}$, while the $D$ 's concentration vanishes at $p_{c 2}$ as $c_{D} \sim\left(p_{c 2}-p\right)^{\beta_{2}}$. In order to justify this behavior a very careful numerical analysis was performed, using longer sampling times in the vicinity of critical points. Fitting the numerical data leads to the above mentioned values of the critical points and $\beta_{1}=\beta_{2}=0.57(3)$ which is compatible with the directed percolation exponent as expected on general ground [20]. This fact is confirmed by the study of concentration fluctuations defined by Eq. (9). Sharp increases of the concentration fluctuations are expected at second order phase transitions. Fig. 9 illustrates this point. The concentration fluctuations of $C$, $D$ and $T$ strategies behave, near the transition point $p_{c 1}$, as $\chi \sim\left(p_{c 1}-p\right)^{-\gamma_{1}}$ with $\gamma_{1}=0.37(6)$. A similar behavior was found at the transition point $p_{c 2}$ with an exponent $\gamma_{2}=0.37(9)$. These values are very close to the one of directed percolation: $\gamma_{D P} \approx 0.35$ in two dimensions [20].

The above data suggest that $p=0$ is another critical point. However, for the reasons explained previously, it was not possible to extract reliable exponents.

It is interesting to analyze how the three strategies coexist for small values of $p$. As an example, let us consider the snapshot of the stationary state of a system with $p=0.04$ (see Fig. 10).

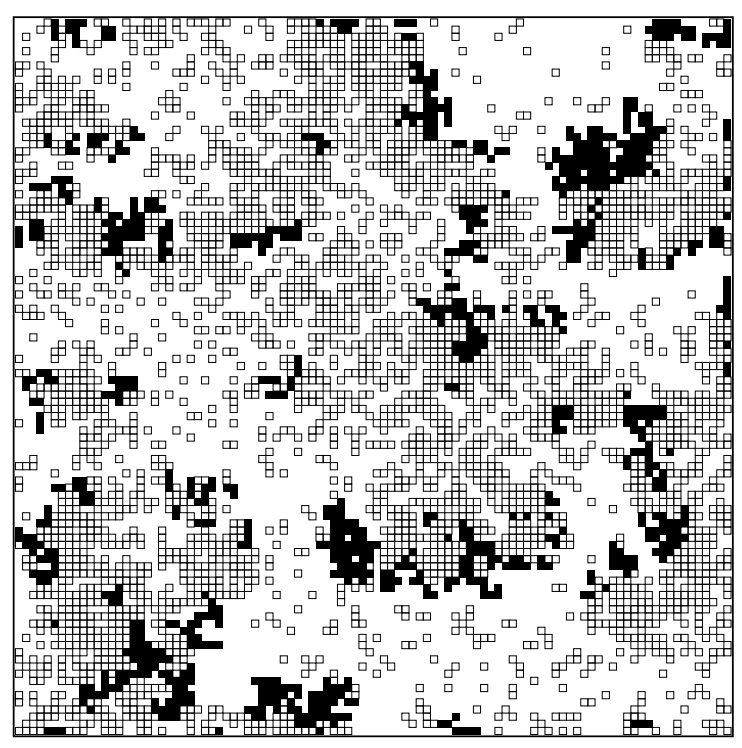

FIG. 10. Distribution of defectors (black boxes), cooperators (white area) and Tit for Tat strategies (open squares) in a system of size $100 \times 100$, subset of a larger system of size $256 \times 256$ for $p=0.04$.

One can observe that "dark areas" (made of defectors) are invading the territory ("white areas") of cooperators, simultaneously, the "dark areas" are invaded by the $T$ actors (open squares). However, the domination of the T's is prevented by the external constraint which is leading to the growing of $C$ areas within the $T$ territory. When $p$ decreases, the $T$ territory expands while the growth of white areas slows down. The dark islands become sparse. On the contrary, an increase of the value of $p$ accelerates the spreading of the $C$ areas as well as their occupation by the defectors. Consequently, the $D$ population increases with $p$ and the number of $T$ competitors decreases and vanishes at $p=p_{c 1}$.

As a result of these cyclic dominant processes a selforganized domain structure is maintained in the system. Analogous spatio-temporal structures have already been observed by Satulowsky and Tomé in a two-dimensional predator-prey system [16] and by Tainaka and Itoh when studying competing species [24]. Both models belong to the family of spatially-extended Lotka-Volterra models predicting oscillatory behavior of concentrations in the simple mean-field approximation 25.

One can also analyze the evolution of the typical strategy configurations in the vicinity of the phase transition taking place at $\left(p=p_{c 1}\right)$. One recognizes isolated colonies of the $T$ strategies whose motions remind us of the branching annihilating random walk models. It is known that this model belongs to the DP universality class too 26.

The rate of mutual cooperation is related to the average payoff per site. The maximum average payoff (which value is 4) is reached when all the players cooperate with their neighbors. On the other hand, the minimum aver- 
age payoff per sites coincides with the maximum of $D$ 's concentration.

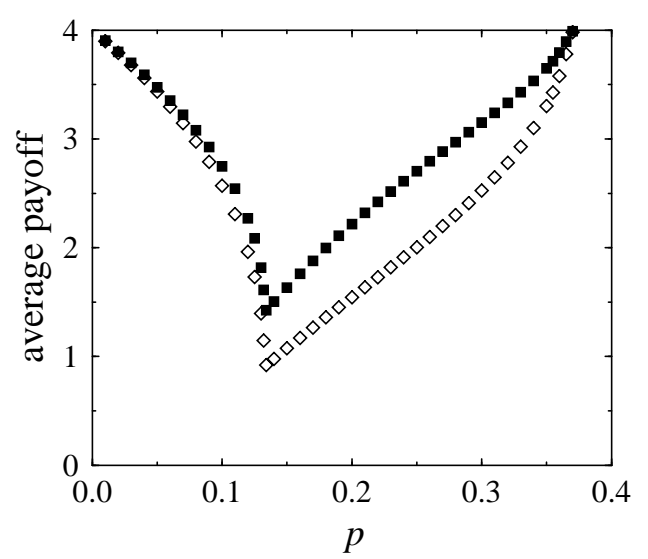

FIG. 11. Average payoff per site sites versus $p$ in the two-dimensional model. The closed squares represent the MC data obtained for $b=1.83$ and $L=512$. The diamonds indicate the quantity $4\left(c_{C}+c_{T}\right)$.

In Fig. 11 the average payoff per site is compared with the quantity $4\left(c_{C}+c_{T}\right)$. One can see that the agreement between these two quantities is generally reasonable and is even quite good for $p<p_{c 1}$. The differences between the two quantities are only coming from the fights taking place at the boundaries $D-C$ or $D-T$. However, for this range of values of $p$, the $D$ players form clusters due to the presence of the $T$ 's and accordingly these fights are not frequent.

Finally, let us briefly consider the case for which the parameter $b$ belongs to the second possible range, $1<$ $b<3 / 2$. The results of the simulations are qualitatively similar to the case $3 / 2<b<2$. The critical values of $p$ are lower, namely, $p_{c 1}=0.112(1)$ and $p_{c 2}=0.297(1)$. The most relevant differences can be observed in the limit $p \rightarrow 0$, where the maximum concentration of the $T$ strategy is strikingly lower $\left(c_{T}<0.15\right)$ than those reported in the previous case (see Fig. 8).

\section{Three-dimensional system}

We now consider the case in which the players are located on the sites of a three-dimensional cubic lattice. According to the payoff matrix (see Table 1), five ranges of values of $b: 1<b<2$ have now to be distinguished, separated by the following values: $b=5 / 4,4 / 3,3 / 2$, and $5 / 3$. Any value of $b$ in one of those ranges will lead strictly to the same behavior. Moreover it turns out that all the values of $b: 1 \leq b \leq 2$ leads qualitatively to the same behavior.

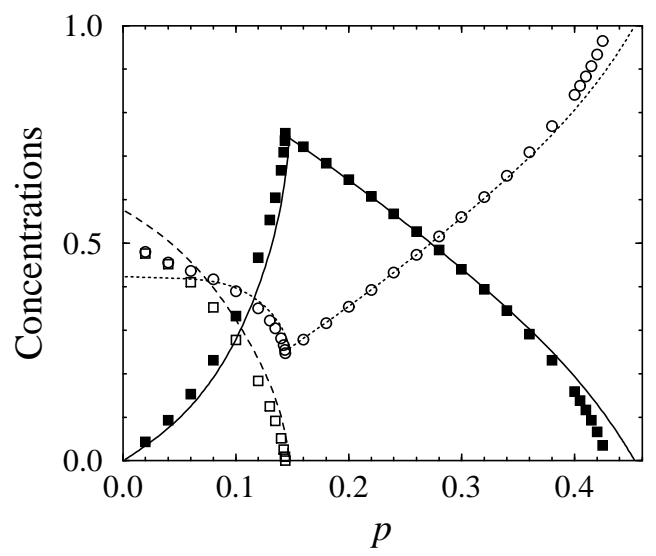

FIG. 12. Stationary state densities of $D$ (closed squares), $C$ (open circles) and $T$ (open squares) strategies as a function of $p$ as obtained by MC simulation of a system of size $80 \times 80 \times 80$. The solid $(D)$, dotted $(C)$ and long-dashed $(T)$ lines indicate the predictions of the pair approximation.

Figure 12 shows a typical phase diagram which is qualitatively similar to the one found for the two-dimensional model. MC simulations have been performed for systems of sizes $32^{3}, 64^{3}$ and $80^{3}$ for five representative values of $b$ exploring all the different ranges.

When the value of $p$ increases, the system undergoes two subsequent phase transitions. The critical values of $p$ vary weakly with $b$. For example, for $3 / 2<b<2$, we have obtained $p_{c 1}^{(M C)}=0.1441(1)$ and $p_{c 2}^{(M C)}=0.4292(5)$, while for $1<b<3 / 2$, we found $p_{c 1}^{(M C)}=0.1512(2)$ and $p_{c 2}^{(M C)}=0.4130(3)$. As expected, the vanishing concentrations behave near the critical points as a power law and both transitions belong to the directed percolation universality class. For $b=1.6$, the numerical analysis of the $\mathrm{MC}$ data in the vicinity of the second transition gives $p_{c 2}^{(M C)}=0.4165(2)$ and $\beta_{2}=0.79(4)$ in good agreement with the corresponding directed percolation value of $\beta=0.81(2)$ 27.

The analysis of the concentration fluctuations $\chi_{T}$ and $\chi_{D}$ in the vicinity of the points $p=p_{c 1}$ and $p=p_{c 2}$ leads to the critical exponent $\gamma=0.18(8)$. The large uncertainty is due to the small extension of the critical regime (typically $\left|p-p_{c}\right| \leq 10^{-3}$ ). The above value of $\gamma$ is compatible with the scaling law for the DP exponents.

As far as mean-field like approximations are concerned, they are supposed to be better when increasing the number of nearest neighbors (dimensions). The algebra becomes soon very cumbersome therefore our analysis is restricted to the pair approximation. The results are given in Fig. 12. One can see that the approximate results are in good agreement with the MC data but in the close vicinity of the critical points. Note finally that the $p$-dependence of the average payoff is qualitatively similar to those found for the two-dimensional model (see Fig. 11). 


\section{CONCLUSIONS}

We have studied quantitatively the emergence of cooperation in a spatially extended version of the prisoner's dilemma game with three possible strategies (cooperation, defection and Tit for Tat) in the absence and presence of externally enforced cooperation. The players are distributed on a $d$-dimensional simple cubic lattice and their interactions are restricted to nearest neighbors.

In the absence of external constraint the time evolution is controlled by a local adoption of a neighboring strategy whose introduction is motivated by the Darwinian selection rule. When starting the simulations with a random initial state made of only cooperators and defectors, one founds that, both in $d=1,2$ and 3 dimensions, the stationary state of the system is a pure defector one. However, if the $T$ strategy is also present in the initial state, then the stationary state is dominated by mutual cooperation.

The external constraint, forcing the players to adopt the strategy $C$ with probability $p$, has the following consequences. If only the $C$ and $D$ strategies are present in the initial state, then the external constraint enforces the cooperation for all value of $p$. However, if the three strategies $C, D$ and $T$ are initially present and if the dimensionality of the system is larger than one, then the external constraint reduces the cooperation maintained by $T$ for small values of $p$. The cooperation will be enforced only if the constraint is large enough $\left(p>p_{c 1}\right)$. These conclusions are reached both from the extended mean-field analysis and the MC simulations for 2 and 3 dimensional systems. The general features are not affected by the value of $b$ characterizing the temptation to defect.

Our study confirms the crucial role of the $T$ strategy which is able to prevent the spreading of defection. The $T$ strategy, however, dyes out in the one-dimensional system as well as in the models for which the simple meanfield theory is exact.

The above conclusions are in agreement with several historical facts coming both from the political or economical world. For example, it shows that forcing a fraction of the population to cooperate in a naive way $(C$ strategy) does not improve the overall cooperation in the society. It is better to educate more individuals in such a way that they will be able to play the more sophisticated Tit for Tat strategy if one desires to improve the cooperation.

From a nonequilibrium phase transition point of view, the above investigations have confirmed that the two second order phase transitions associated with the extinction processes belong to the robust directed percolation universality class.

Finally we emphasize that similar behavior is expected for spatially extended Lotka-Volterra like systems with three (or more) species providing that where one of the species is externally favored.

\section{ACKNOWLEDGMENTS}

We thank Gunter Schütz for a very helpful discussion. This work was supported by the Hungarian National Research Fund under Grant No. T-23552 and by the Swiss National Foundation.

[1] J. Maynard Smith, Evolution and the Theory of Games (Cambridge University Press, Cambridge, 1982).

[2] J. Hofbauer and K. Sigmund, Evolutionary Games and Population Dynamics (Cambridge University Press, Cambridge, England, 1998).

[3] K. Sigmund, Games of Life, (Oxford University Press, Oxford, 1993).

[4] R. Axelrod, The Evolution of Cooperation (Basic Books, New York, 1984).

[5] J. W. Weibull, Evolutionary Game Theory (MIT Press, Cambridge, 1995).

[6] M. A. Nowak and R. M. May, Int. J. Bifur. Chaos 3, 35 (1993); A. Novak and R. M. May, Nature 359, 826-829 (1992).

[7] J.-P Delahaye, Dossier "Pour la Science", 58 ( July 1999).

[8] T. Killingback, M. Doebeli and N. Knowlton, Proc. R. Soc. Lond. B266, 1723-1728 (1999).

[9] T. Killingback and M. Doebeli, Proc. R. Soc. Lond. B263, 1135-1144 (1996).

[10] T. Killingback and M. Doebeli, J. theor. Biol. 200, 405417 (1999).

[11] K. Brauchli, T. Killingback and M. Doebeli, J. theor. Biol. 191, 335-340 (1998).

[12] M. A. Nowak, S. Bonhoeffer, and R. M. May, Int. J. Bifur. Chaos 4, 33 (1993).

[13] G. Szabó and C. Tőke, Phys. Rev. E 53, 2196 (1998).

[14] J. R. N. Chiappin and M. J. de Oliveira, Phys. Rev. E 59, 6419 (1999).

[15] H. A. Gutowitz, J. D. Victor, and B. W. Knight, Physica 28D, 18 (1987); G. Szabó, A. Szolnoki, and L. Bodócs, Phys. Rev. A 44, 6375 (1991); G. Szabó and G. Ódor, Phys. Rev. E 49, 2764 (1994).

[16] J. E. Satulowsky and T. Tomé, Phys. Rev. E 49, 5073 (1994).

[17] T. E. Harris, Ann. Prob. 2, 969 (1974)

[18] I. Jensen and R. Dickman, J. Stat. Phys. 71, 89 (1993)

[19] H. K. Janssen, Z. Phys. B 42, 151 (1981); P. Grassberger, Z. Phys. B 47, 365 (1982).

[20] C. Brower, M. A. Furman, and M. Moshe, Phys. Lett. 76B, 213 (1978); I. Jensen, H. C. Fogedby, and R. Dickman, Phys. Rev. A 41, 3411 (1990).

[21] Very recently Frachebourg et al have studied similar processes in a simplified model where the cyclic symmetry has provided that the fronts travels (left or right) with the same average velocity: L. Frachebourg, P. L. Krapivsky, and E. Ben-Naim, Phys. Rev. E 54, 6186 (1996).

[22] P. Grassberger and H. Kantz, J. Stat. Phys. 63, 685 (1991); B. Drossel and F. Schwabl, Phys. Rev. Lett. 
69, 1629 (1992); J. E. S. Socolar, G. Grinstein, and C. Jayaprakash, Phys. Rev. E 47, 2366 (1993); H.-M. Bröker and P. Grassberger, Phys. Rev. E 56, R4918 (1997).

[23] P. Bak, K. Chen, and C. Tang, Phys. Lett. A 147, 297 (1990).

[24] K. Tainaka and Y. Itoh, Europhys. Lett. 15, 399 (1991).

[25] A. J. Lotka, Proc. Natl. Acad. Sci. U.S.A. 6, 410 (1920); V. Volterra, Leçon sur la Theorie Mathematique de la Lutte pour le Vie (Gauthier-Villars, Paris, 1931).

[26] J. Cardy and U. C. Täuber, Phys. Rev. Lett. 77, 4780 (1996); J. Stat. Phys. 90, 1 (1998); and further references therein.

[27] I. Jensen, Phys. Rev. A 45, R563 (1992). 